\title{
Multi-Band Intra-Night Optical Variability of BL Lacertae
}

\author{
Haritma Gaur ${ }^{1, *}$, Alok C. Gupta ${ }^{2}$, Rumen Bachev ${ }^{3}$, Anton Strigachev ${ }^{3}$, Evgeni Semkov ${ }^{3}$, \\ Paul J. Wiita ${ }^{4,5}$, Minfeng Gu ${ }^{1}$ and Sunay Ibryamov 6 \\ 1 Key Laboratory for Research in Galaxies and Cosmology, Shanghai Astronomical Observatory, \\ Chinese Academy of Sciences, 80 Nandan Road, Shanghai 200030, China; gumf@shao.ac.cn \\ 2 Aryabhatta Research Institute of Observational Sciences (ARIES), Manora Peak, Nainital 263002, India; \\ acgupta30@gmail.com \\ 3 Institute of Astronomy and National Astronomical Observatory, Bulgarian Academy of Sciences, \\ 72 Tsarigradsko Shosse Blvd., 1784 Sofia, Bulgaria; blazonstone@gmail.com (R.B); \\ anton.strigachev@gmail.com (A.S); esemkov@astro.bas.bg (E.S) \\ 4 Department of Physics, The College of New Jersey, P.O. Box 7718, Ewing, NJ 08628-0718, USA; \\ wiitap@tcnj.edu \\ 5 Kavli Institute for Particle Astrophysics and Cosmology, SLAC, 2575 Sand Hill Rd, Menlo Park, \\ CA 94025, USA \\ 6 Department of Theoretical and Applied Physics, Faculty of Natural Sciences, University of Shumen, 115, \\ Universitetska Str., 9712 Shumen, Bulgaria; sibryamov@shu.bg \\ * Correspondence: harry.gaur31@gmail.com
}

Received: 30 October 2017; Accepted: 5 December 2017; Published: 8 December 2017

\begin{abstract}
We monitored BL Lacertae frequently during 2014-2016 when it was generally in a high state. We searched for intra-day variability for 43 nights using quasi-simultaneous measurements in the $\mathrm{B}, \mathrm{V}, \mathrm{R}$, and I bands (totaling 143 light curves); the typical sampling interval was about eight minutes. On hour-like timescales, BL Lac exhibited significant variations during 13 nights in various optical bands. Significant spectral variations are seen during most of these nights such that the optical spectrum becomes bluer when brighter. The amplitude of variability is usually greater for longer observations but is lower when BL Lac is brighter. No evidence for periodicities or characteristic variability time-scales in the light curves was found. The color variations are mildly chromatic on long timescales.
\end{abstract}

Keywords: active galaxies; BL Lacertae object: BL Lac; jets

\section{Introduction}

BL Lacertae is a prototype of the blazar class at redshift $z=0.069$ [1]. BL Lacs, along with many flat spectrum radio quasars, constitute the blazar class of active galaxies. BL Lacs are differentiated by the absence or extreme weakness of emission lines (usually taken to be an equivalent width in the rest frame of the host galaxy of $<5 \AA$ ). They exhibit strong flux and spectral variability across all of the electromagnetic spectrum over a wide range of time-scales, and significant and variable radio and optical polarization is common to all blazars (e.g., [2]). However, on some occasions, BL Lacertae has shown broad $H \alpha$ and $H \beta$ emission lines in its spectrum, thereby raising the issue of it actually being part of its eponymous class [3]. BL Lac is classified as a low frequency peaked blazar (LBL) because of its first spectral hump peaks in the near IR/optical region, which is explained as the synchrotron emission from highly relativistic electrons within a helical jet [4]. Optical observations are particularly helpful in studying the acceleration and cooling timescales of the electrons in the relativistic jets.

BL Lacertae has shown strong variability in optical bands and hence is one of the favorite targets of multi-wavelength campaigns carried out by the Whole Earth Blazar Telescope (WEBT/GASP; [4-8] and 
references therein). BL Lac displays intense optical variability on short and intra-day time-scales (e.g., [9-13]) as well as substantial variations in its polarization ( $[14,15]$ and references therein). Previous studies have stressed the flux variations and spectral changes ( [16-18]).

In this work, we present observations of BL Lac, made at several telescopes in Bulgaria and Greece, during the period 2014-2016 over a total of 43 nights with sufficient measurements so as to be able to properly search for intra-night optical variability. A key motivation for this study is to understand the nature of flux and spectral variability of BL Lac on the shortest intra-day timescales. This study is a follow up of our earlier extensive optical monitoring of BL Lacertae covering the period 2010-2012 ( $[19,20])$. These additional observations of this particular blazar help us to better constrain its duty cycle for hourly variability in optical bands and let us study the dependences of the amplitude of variability on the overall flux levels. In Section 2, we briefly describe our observations and data reductions. Section 3 summarizes the methods we used to quantify variability. We present our results in Section 4. Sections 5 and 6, respectively, give a discussion and our conclusions.

\section{Observations and Data Reduction}

Our new observations of BL Lacertae began on 29 July 2014 and concluded on 30 September 2016 continuing those taken between 10 June 2010 and 26 October 2012 that we reported in [19]. The complete log of observations is given in Table 1. Our observations were made using two telescopes in Bulgaria and Greece. Details about the telescopes in Bulgaria and Greece are given in ( [21], Table 1). The standard data reduction methods we employed on all observations are described in detail in Section 3 of [21]. Typical seeing varied between 1-3 arcsec during these measurements.

To summarize, standard data reduction was performed using IRAF $^{1}$, including bias subtraction and flat-field division. Instrumental magnitudes of BL Lacertae as well as four comparison stars in its field [22] were produced using the IRAF package DAOPHOT ${ }^{2}$; normally, an aperture radius of $8^{\prime \prime}$ was used.

In these measurements of BL Lacertae's light curve (LC), we observe comparison stars B, C, and H in the same field and take their magnitudes from [22]. Star $C$ is used for calibration because both its magnitude and color were most similar to those of BL Lac during this period. BL Lac is within a relatively bright host galaxy and we removed its contribution from the observed magnitudes by following the method provided in [19].

Table 1. Observation log of BL Lacertae.

\begin{tabular}{cccc}
\hline $\begin{array}{c}\text { Date of Observation } \\
\text { dd.mm.yyyy }\end{array}$ & Telescope & Band & $\begin{array}{c}\text { Number of } \\
\text { Image Frames }\end{array}$ \\
\hline 29.07 .2014 & A & B, V, R, I & $10,10,10,10$ \\
28.09 .2014 & B & B, V, R, I & $13,17,19,23$ \\
29.09 .2014 & B & B, V, R, I & $12,16,17,19$ \\
30.09 .2014 & B & B, V, R, I $19,20,22,23$ \\
29.06 .2015 & A & B, V, R, I $10,10,10,10$ \\
30.06 .2015 & A & B, V, R, I $10,10,10,10$ \\
18.07 .2015 & B & B, R, I & $33,33,32$ \\
19.07 .2015 & B & B, R, I & $26,27,27$ \\
20.07 .2015 & B & B, R, I & $22,24,24$ \\
\hline
\end{tabular}

1 IRAF is distributed by the National Optical Astronomy Observatories, which are operated by the Association of Universities for Research in Astronomy, Inc., Cranford, NJ, USA, under cooperative agreement with the National Science Foundation.

2 Dominion Astrophysical Observatory Photometry software 
Table 1. Cont.

\begin{tabular}{cccc}
\hline $\begin{array}{c}\text { Date of Observation } \\
\text { dd.mm.yyyy }\end{array}$ & Telescope & Band & $\begin{array}{c}\text { Number of } \\
\text { Image Frames }\end{array}$ \\
\hline 21.07 .2015 & B & B, R, I & $20,20,20$ \\
22.07 .2015 & B & B, R, I & $23,24,24$ \\
23.07 .2015 & B & B, R, I & $18,22,20$ \\
18.08 .2015 & B & B, R, I & $28,28,28$ \\
26.08 .2015 & B & B, R, I & $17,20,17$ \\
27.08 .2015 & B & B, R, I & $19,21,20$ \\
28.08 .2015 & B & B, R, I & $22,23,21$ \\
08.09 .2015 & B & B, R, I & $45,45,45$ \\
14.09 .2015 & B & B, R, I & $23,27,29$ \\
15.09 .2015 & B & B, R, I & $28,28,30$ \\
03.11 .2015 & B & B, R, I & $21,21,22$ \\
04.11 .2015 & B & B, R, I & $26,28,28$ \\
05.11 .2015 & B & B, R, I & $30,30,30$ \\
06.11 .2015 & B & B, R, I & $31,33,32$ \\
07.11 .2015 & B & B, R, I & $28,32,32$ \\
11.11 .2015 & B & B, R, I & $46,46,45$ \\
12.11 .2015 & B & B, R, I & $43,42,41$ \\
03.12 .2015 & B & B, R, I & $15,16,18$ \\
14.12 .2015 & B & B, R, I & $29,30,30$ \\
30.06 .2016 & B & V, R, I & $20,20,20$ \\
01.07 .2016 & B & V, R, I & $14,14,13$ \\
08.08 .2016 & B & B, V, R & $15,15,30$ \\
09.08 .2016 & B & B, V, R & $25,25,51$ \\
25.08 .2016 & B & B, V, R & $13,14,28$ \\
26.08 .2016 & B & B, V, R & $25,26,59$ \\
28.08 .2016 & B & B, V, R, I & $14,14,12,13$ \\
30.08 .2016 & B & B, V, R, I & $45,54,53,53$ \\
23.09 .2016 & B & B, V, R, I & $44,44,42,23$ \\
24.09 .2016 & B & B, V, R, I & $31,30,30,22$ \\
26.09 .2016 & B & B, V, R, I & $45,45,43,42$ \\
27.09 .2016 & B & B, V, R, I & $42,42,41,37$ \\
28.09 .2016 & B & B, V, R, I & $50,46,47,49$ \\
29.09 .2016 & B & B, V, R, I & $49,49,50,42$ \\
30.09 .2016 & B & B, V, R, I & $38,35,34,36$ \\
\hline
\end{tabular}

A: 1.3-m Skinakas Observatory, Crete, Greece; B: 60-cm Cassegrain Telescope at Astronomical Observatory Belogradchik, Bulgaria.

\section{Variability Detection Criterion}

The $F$-test is considered to be a sensible way to search for the presence of the intra-night variability and has frequently been used to do so (i.e., [23]). However, recently, Reference [24] pointed out a problem with this apparently robust method, showing that either substantial brightness differences or significant variability in a comparison star can produce underestimation of the source variability with respect to the dimmer or less variable star.

To avoid these problems, we employed the power-enhanced $F$-test ( $[24,25])$. The basic idea is that one first increases the number of degrees of freedom in the denominator of the $F$-distribution by stacking all the LCs of the standard stars. Next, the comparison star differential LCs are transformed so as to have the same photometric noise as the blazar. The key point is that, by using multiple comparison stars, the probability of false claims of intra-night variability are reduced compared to when only a single standard star is used. See [25] for a detailed discussion.

The smaller the $\alpha$ value, the more improbable it is that the result is produced by chance. If $F$ exceeds the critical value, the no variability (null) hypothesis should be discarded. We use a stringent level of $\alpha=0.001$ and have performed the power enhanced $F$-test on all those LCs where at least ten points are available. 


\section{Results}

\subsection{Intra-Day Variability}

Our extensive observations of BL Lacertae were made on 43 nights during 2014-2016. During those nights, BL Lac was measured for brightness quasi-simultaneously in at least two, and usually at least three, of the B, V, R and I bands, yielding a total of 143 LCs. These LCs are displayed in Figures 1 and 2 and show that the R-band brightnesses ranged between 13.4 and 12.3 mag but were almost always brighter than $m=13$. Our nightly observations mostly spanned between 2 and $5 \mathrm{~h}$, with typical intervals between exposures in a given band being $8 \mathrm{~min}$.

It can be seen from the figures that BL Lac was variable on intra-day timescales and often showed some variability on hourly time-scales. We used the enhanced F-test to search for genuine flux variations on these intra-day variability (IDV) time-scales, finding 19 LCs to be variable across the filter set over the course of 13 nights of observation. Of these, 11 were seen in the $\mathrm{R}$ band, for which we have measurements on every night we observed and which also have the smallest errors. These enhanced F-test values are presented in Table 2. We note that only rarely did more than one band show strong enough variability to satisfy the stringent criterion during the same nights, mostly because of different error levels in the different bands. In particular, the B band errors are usually significantly greater than those at other bands and essentially preclude our verifying any but the most extreme variations. The nightly LCs displayed rises and decays up to a magnitude change of $\sim 0.18$ over several hours. A few such examples of substantial variations are shown with expanded time axes in Figure 3. In most of the observations, flickering/small subflares are superimposed on multi-hour trends that are presumably parts of larger flares.

The duty cycle (DC, in percent) of BL Lac was found using the definition of [26] that subsequently was frequently employed (e.g., [19,27] and references therein).

Given the multi-band data we collected, we can compute the DC in different ways. If all detections of variability are considered, we have 19 clearly variable LCs out of 143 in total, producing a DC of around $12 \%$. If we exclude the noisy B band, we have 17 variable LCs out of 102 . Perhaps the fairest approach is to only consider the most accurate $\mathrm{R}$ band data, and most previous studies have just looked at one band (usually R): in this case, we have firm detections on 11 out of 43 nights, or a DC of roughly $25 \%$.

In our previous extensive search for IDV in BL Lac over 38 nights in the period 2010-2012, we found 50 (out of 119) filter LCs to be variable during 19 nights using the same enhanced F-test [19], or a $\mathrm{DC}=44 \%$, although the B band data were usually better then. If we restrict our computation to only $\mathrm{R}$ band data, we find $\mathrm{DC}=49 \%$ in this earlier period. We note that BL Lac had a lower average brightness during 2010-2012, with R-band magnitudes ranging between 13.9 and 12.4 and it was usually fainter than $m=13$. These differences in the duty cycle between these two periods could be attributed to a reduction in the turbulence behind a standing shock in the jet, which is a leading explanation for rapid, intra-day variability in blazars (e.g., [28-30]). This turbulence may decrease as the bulk jet speed rises and so the overall observed source flux increases. Hence, it is reasonable to expect to see a comparatively lower DC when the source becomes brighter.

The percentage variability amplitudes are calculated using the standard method of [31]. It has been found in some previous studies of BL Lacs that the variability amplitude is larger at higher frequencies, specifically for B (and occasionally V), on nights when variability is certainly detected in the $\mathrm{R}$ and I bands [9,32,33]; however, in other studies, the strength of the variability was found to not be systematically larger at higher frequencies ( $[34,35])$. In our current observations, the B band errors are significantly higher as compared to lower frequency bands, so that, on many occasions, our significance thresholds in B (also sometimes V) exceed the apparent fluctuations, even when $\mathrm{R}$ band variability is clearly seen and is statistically significant. The amplitude of variability in nights with clear detections varied between $7-20 \%$ in the R band; however, the highest fractional amplitude 
of variability was seen for the night of 26 September 2016, where an amplitude of $41 \%$ was measured for the B band.
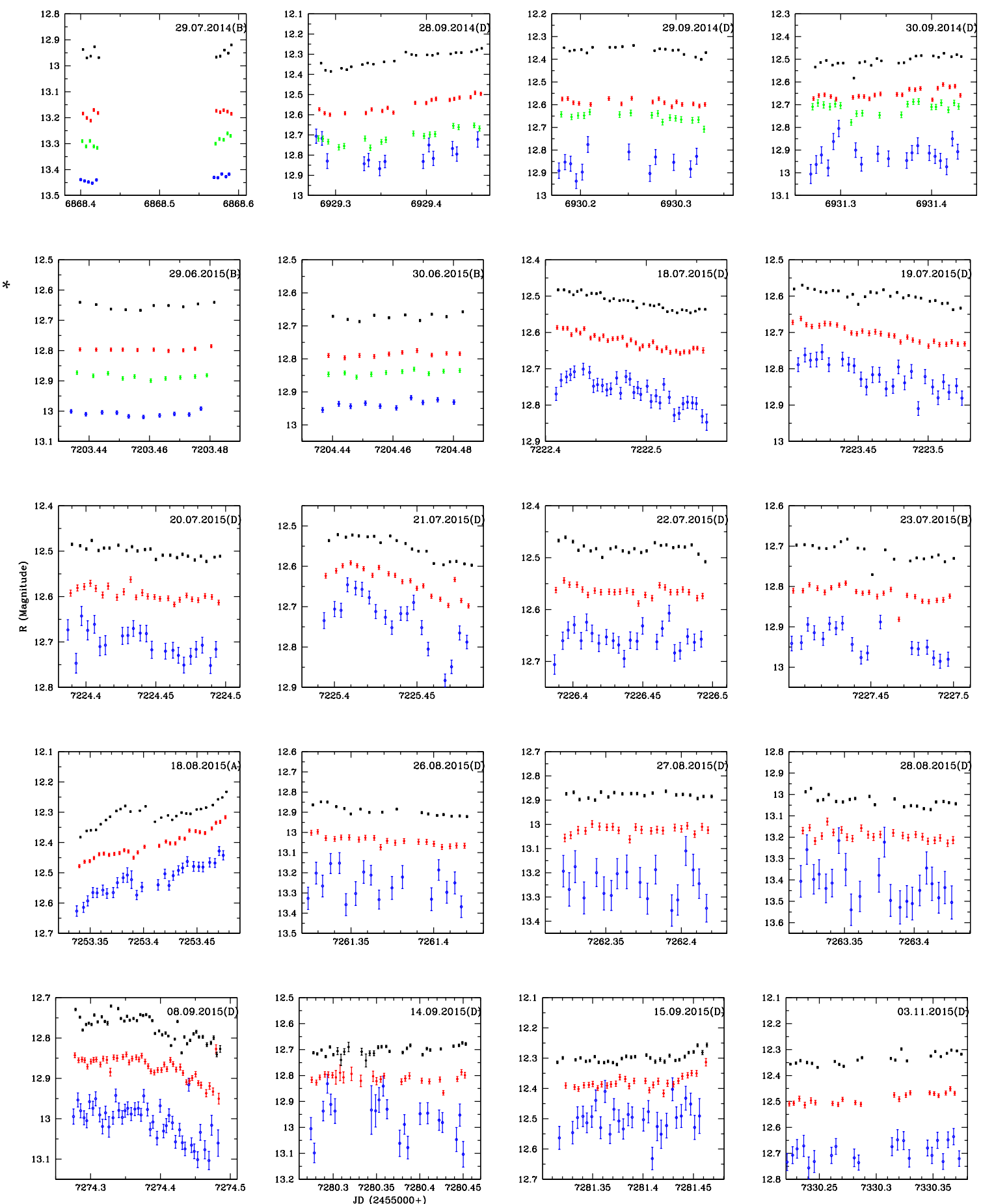

Figure 1. IDV LCs for BL Lacertae from July 2014 through November 2015 in the B (blue), V (green), $\mathrm{R}$ (red) and I (black) bands. The $x$-axis is JD (2455000+), and the $y$-axis is the calibrated R band magnitudes in each of the panels. For clarity, the B, V and I LCs are shifted by arbitrary offsets with respect to the R-band LC. 

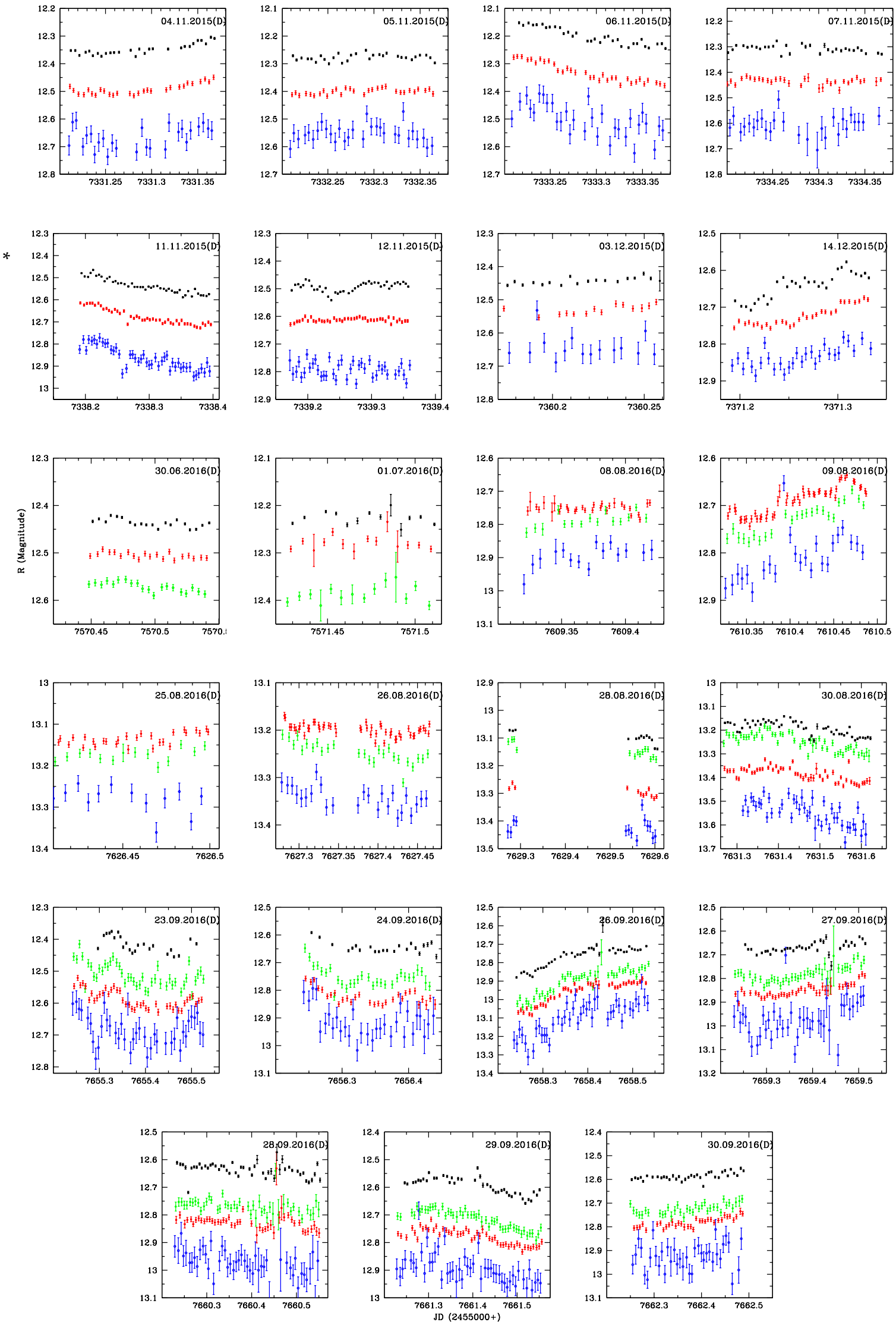

Figure 2. As in Figure 1, for November 2015 through September 2016. 
The correlation between the variability strength and source flux is shown in Figure 4; we see that the variability amplitude drops as the source flux rises $(\rho=-0.42$ with $p=0.9996$ where $\rho$ is the Spearman correlation coefficient). Here, we included our data from [19] for the 2010-2012 period. As for our above discussion of the DC, this trend can be explained if, as the source's flux increases, the irregularities in the jet flow, i.e., turbulence near shocks/knots, decrease. As this turbulence is quite likely to be responsible for the intra-night optical variability ( [28-30]), an overall increased flux rise would lead to a reduction in the fractional amplitude (e.g., [36]). Part of the scatter in the plot could arise because observed amplitudes of variability seen for similar luminosities normally go up for longer observations, and there is a significant spread in the observation times in our data.

Table 2. Results of intra-day variability.

\begin{tabular}{|c|c|c|c|c|c|c|}
\hline $\begin{array}{c}\text { Date of Observation } \\
\text { dd.mm.yyyy }\end{array}$ & Band & Number & $F$ & $F_{c}(0.001)$ & Variability & Amplitude \\
\hline \multirow[t]{4}{*}{29.07 .2014} & B & 10 & 4.66 & 5.56 & NV & - \\
\hline & $\mathrm{V}$ & 10 & 3.54 & 5.56 & NV & - \\
\hline & $\mathrm{R}$ & 10 & 2.81 & 5.56 & NV & - \\
\hline & I & 10 & 1.75 & 5.56 & NV & - \\
\hline \multirow[t]{4}{*}{28.09 .2014} & B & 13 & 0.858 & 4.393 & $\mathrm{NV}$ & - \\
\hline & V & 17 & 1.115 & 3.598 & $\mathrm{NV}$ & - \\
\hline & $\mathrm{R}$ & 19 & 1.769 & 3.345 & NV & - \\
\hline & I & 23 & 1.084 & 2.983 & NV & - \\
\hline \multirow[t]{4}{*}{29.09 .2014} & B & 12 & 0.345 & 4.697 & NV & - \\
\hline & $\mathrm{V}$ & 16 & 0.277 & 3.753 & NV & - \\
\hline & $\mathrm{R}$ & 17 & 0.346 & 3.598 & NV & - \\
\hline & I & 19 & 0.232 & 3.345 & NV & - \\
\hline \multirow[t]{4}{*}{30.09 .2014} & B & 19 & 0.701 & 3.345 & NV & - \\
\hline & $\mathrm{V}$ & 20 & 0.492 & 3.239 & NV & - \\
\hline & $\mathrm{R}$ & 22 & 0.246 & 3.06 & NV & - \\
\hline & I & 23 & 0.355 & 2.983 & NV & - \\
\hline \multirow[t]{4}{*}{29.06 .2015} & B & 10 & 2.10 & 5.56 & NV & - \\
\hline & $\mathrm{V}$ & 10 & 2.00 & 5.56 & NV & - \\
\hline & $\mathrm{R}$ & 10 & 3.19 & 5.56 & $\mathrm{NV}$ & - \\
\hline & I & 10 & 2.32 & 5.56 & NV & - \\
\hline \multirow[t]{4}{*}{30.06 .2015} & B & 10 & 2.08 & 5.56 & $\mathrm{NV}$ & - \\
\hline & $\mathrm{V}$ & 10 & 2.14 & 5.56 & NV & - \\
\hline & $\mathrm{R}$ & 10 & 2.76 & 5.56 & NV & - \\
\hline & I & 10 & 1.15 & 5.56 & NV & - \\
\hline \multirow[t]{3}{*}{ 18.07.2015 } & B & 33 & 0.327 & 2.481 & NV & - \\
\hline & $\mathrm{R}$ & 33 & 4.549 & 2.481 & Var & 7.14 \\
\hline & I & 32 & 2.252 & 2.517 & NV & - \\
\hline \multirow[t]{3}{*}{ 19.07.2015 } & B & 26 & 0.812 & 2.79 & NV & - \\
\hline & $\mathrm{R}$ & 27 & 3.973 & 2.736 & Var & 7.57 \\
\hline & I & 27 & 0.881 & 2.736 & $\mathrm{NV}$ & - \\
\hline \multirow[t]{3}{*}{20.07 .2015} & B & 22 & 0.200 & 3.06 & NV & - \\
\hline & $\mathrm{R}$ & 24 & 1.221 & 2.913 & NV & - \\
\hline & I & 24 & 0.742 & 2.913 & NV & - \\
\hline \multirow[t]{3}{*}{ 21.07.2015 } & B & 20 & 1.086 & 3.239 & NV & - \\
\hline & $\mathrm{R}$ & 20 & 6.851 & 3.239 & Var & 10.67 \\
\hline & I & 20 & 2.226 & 3.239 & NV & - \\
\hline \multirow[t]{3}{*}{ 22.07.2015 } & B & 23 & 0.373 & 2.983 & NV & - \\
\hline & $\mathrm{R}$ & 24 & 0.669 & 2.913 & NV & - \\
\hline & I & 24 & 0.320 & 2.913 & NV & - \\
\hline
\end{tabular}


Table 2. Cont.

\begin{tabular}{|c|c|c|c|c|c|c|}
\hline $\begin{array}{c}\text { Date of Observation } \\
\text { dd.mm.yyyy }\end{array}$ & Band & Number & $F$ & $F_{c}(0.001)$ & Variability & Amplitude \\
\hline \multirow[t]{3}{*}{23.07 .2015} & B & 18 & 0.460 & 3.463 & NV & - \\
\hline & $\mathrm{R}$ & 22 & 1.337 & 3.06 & NV & - \\
\hline & $\mathrm{I}$ & 20 & 0.853 & 3.239 & NV & - \\
\hline \multirow[t]{3}{*}{18.08 .2015} & B & 28 & 1.355 & 2.686 & $\mathrm{NV}$ & - \\
\hline & $\mathrm{R}$ & 28 & 13.825 & 2.686 & Var & 16.15 \\
\hline & I & 28 & 1.924 & 2.686 & NV & - \\
\hline \multirow[t]{3}{*}{26.08 .2015} & B & 17 & 0.488 & 3.598 & $\mathrm{NV}$ & - \\
\hline & $\mathrm{R}$ & 20 & 6.659 & 3.239 & Var & 7.67 \\
\hline & I & 17 & 1.125 & 3.598 & NV & - \\
\hline \multirow[t]{3}{*}{ 27.08.2015 } & B & 19 & 0.335 & 3.345 & $\mathrm{NV}$ & - \\
\hline & $\mathrm{R}$ & 21 & 0.669 & 3.145 & NV & - \\
\hline & I & 20 & 0.336 & 3.239 & NV & - \\
\hline \multirow[t]{3}{*}{ 28.08.2015 } & B & 22 & 0.633 & 3.06 & $\mathrm{NV}$ & - \\
\hline & $\mathrm{R}$ & 23 & 3.093 & 2.983 & Var & 10.16 \\
\hline & $\mathrm{I}$ & 21 & 1.797 & 3.145 & NV & - \\
\hline \multirow[t]{3}{*}{08.09 .2015} & B & 45 & 0.928 & 2.176 & $\mathrm{NV}$ & - \\
\hline & $\mathrm{R}$ & 45 & 1.249 & 2.176 & NV & - \\
\hline & I & 45 & 1.233 & 2.176 & NV & - \\
\hline \multirow[t]{3}{*}{14.09 .2015} & B & 23 & 0.422 & 2.983 & NV & - \\
\hline & $\mathrm{R}$ & 27 & 0.147 & 2.736 & NV & - \\
\hline & I & 29 & 0.16 & 2.639 & NV & - \\
\hline \multirow[t]{3}{*}{15.09 .2015} & B & 28 & 0.213 & 2.686 & NV & - \\
\hline & $\mathrm{R}$ & 28 & 0.163 & 2.686 & NV & - \\
\hline & I & 30 & 0.121 & 2.596 & NV & - \\
\hline \multirow[t]{2}{*}{03.11 .2015} & B & 21 & 0.978 & 3.145 & NV & - \\
\hline & $\mathrm{R}$ & 21 & 0.734 & 3.145 & $\mathrm{NV}$ & - \\
\hline \multirow{3}{*}{04.11 .2015} & I & 22 & 0.508 & 3.06 & $\mathrm{NV}$ & - \\
\hline & B & 26 & 0.568 & 2.79 & NV & - \\
\hline & $\mathrm{R}$ & 28 & 2.599 & 2.686 & $\mathrm{NV}$ & - \\
\hline \multirow{4}{*}{05.11 .2015} & I & 28 & 0.749 & 2.686 & $\mathrm{NV}$ & - \\
\hline & B & 30 & 0.568 & 2.596 & NV & - \\
\hline & $\mathrm{R}$ & 30 & 1.559 & 2.596 & NV & - \\
\hline & I & 30 & 0.833 & 2.596 & NV & - \\
\hline \multirow[t]{3}{*}{06.11 .2015} & B & 31 & 0.88 & 2.555 & $\mathrm{NV}$ & - \\
\hline & $\mathrm{R}$ & 33 & 6.93 & 2.481 & Var & 10.57 \\
\hline & I & 32 & 3.411 & 2.517 & Var & 9.33 \\
\hline \multirow[t]{2}{*}{ 07.11.2015 } & B & 28 & 0.245 & 2.686 & NV & - \\
\hline & $\mathrm{R}$ & 32 & 0.39 & 2.517 & $\mathrm{NV}$ & - \\
\hline \multirow{3}{*}{11.11 .2015} & I & 32 & 0.096 & 2.517 & NV & - \\
\hline & B & 46 & 0.764 & 2.157 & NV & - \\
\hline & $\mathrm{R}$ & 46 & 6.571 & 2.157 & Var & 11.30 \\
\hline \multirow{4}{*}{ 12.11.2015 } & I & 45 & 8.000 & 2.176 & Var & 12.39 \\
\hline & B & 43 & 1.212 & 2.215 & NV & - \\
\hline & $\mathrm{R}$ & 42 & 0.369 & 2.236 & NV & - \\
\hline & I & 41 & 0.879 & 2.258 & NV & - \\
\hline \multirow[t]{3}{*}{03.12 .2015} & B & 15 & 1.273 & 3.932 & $\mathrm{NV}$ & - \\
\hline & $\mathrm{R}$ & 16 & 1.111 & 3.753 & NV & - \\
\hline & I & 18 & 0.558 & 3.463 & NV & - \\
\hline \multirow[t]{3}{*}{14.12 .2015} & B & 29 & 0.512 & 2.639 & NV & - \\
\hline & $\mathrm{R}$ & 30 & 7.858 & 2.596 & Var & 8.31 \\
\hline & I & 30 & 2.843 & 2.596 & Var & 13.09 \\
\hline
\end{tabular}


Table 2. Cont.

\begin{tabular}{|c|c|c|c|c|c|c|}
\hline $\begin{array}{c}\text { Date of Observation } \\
\text { dd.mm.yyyy }\end{array}$ & Band & Number & $F$ & $F_{c}(0.001)$ & Variability & Amplitude \\
\hline & $\mathrm{R}$ & 20 & 1.081 & 3.239 & NV & - \\
\hline & $\mathrm{I}$ & 20 & 0.09 & 3.239 & NV & - \\
\hline & $\mathrm{R}$ & 14 & 0.708 & 4.142 & NV & - \\
\hline & I & 13 & 0.839 & 4.393 & NV & - \\
\hline \multirow[t]{3}{*}{08.08 .2016} & B & 15 & 0.607 & 3.932 & NV & - \\
\hline & $\mathrm{V}$ & 15 & 1.57 & 3.932 & NV & - \\
\hline & $\mathrm{R}$ & 30 & 0.467 & 2.596 & NV & - \\
\hline \multirow{3}{*}{ 09.08.2016 } & B & 25 & 2.594 & 2.849 & NV & - \\
\hline & V & 25 & 4.572 & 2.849 & Var & 22.54 \\
\hline & $\mathrm{R}$ & 51 & 1.915 & 2.076 & NV & - \\
\hline \multirow[t]{3}{*}{25.08 .2016} & B & 13 & 0.930 & 4.393 & NV & - \\
\hline & $\mathrm{V}$ & 14 & 0.791 & 4.142 & NV & - \\
\hline & $\mathrm{R}$ & 28 & 1.290 & 2.686 & NV & - \\
\hline \multirow[t]{3}{*}{26.08 .2016} & B & 25 & 0.403 & 2.849 & $\mathrm{NV}$ & - \\
\hline & $\mathrm{V}$ & 26 & 0.526 & 2.79 & NV & \\
\hline & $\mathrm{R}$ & 59 & 0.942 & 1.972 & NV & - \\
\hline \multirow[t]{3}{*}{28.08 .2016} & B & 14 & 0.698 & 4.142 & NV & - \\
\hline & $\mathrm{R}$ & 12 & 1.009 & 4.697 & NV & - \\
\hline & I & 13 & 1.632 & 4.393 & NV & - \\
\hline \multirow[t]{3}{*}{30.08 .2016} & B & 45 & 2.218 & 2.176 & Var & 21.41 \\
\hline & $\mathrm{V}$ & 54 & 2.498 & 2.033 & Var & 12.73 \\
\hline & $\mathrm{R}$ & 53 & 1.408 & 2.047 & NV & - \\
\hline \multirow{3}{*}{23.09 .2016} & I & 53 & 1.775 & 2.047 & $\mathrm{NV}$ & - \\
\hline & B & 44 & 1.023 & 2.195 & NV & - \\
\hline & $\mathrm{V}$ & 44 & 1.998 & 2.195 & NV & - \\
\hline \multirow{6}{*}{24.09 .2016} & $\mathrm{R}$ & 42 & 3.48 & 2.236 & Var & 10.75 \\
\hline & I & 23 & 2.834 & 2.983 & NV & \\
\hline & B & 31 & 0.642 & 2.555 & NV & - \\
\hline & $\mathrm{V}$ & 30 & 1.433 & 2.596 & NV & - \\
\hline & $\mathrm{R}$ & 30 & 2.054 & 2.596 & NV & - \\
\hline & I & 22 & 0.617 & 3.06 & NV & - \\
\hline \multirow[t]{4}{*}{26.09 .2016} & B & 45 & 3.496 & 2.176 & Var & 40.87 \\
\hline & $\mathrm{V}$ & 45 & 6.274 & 2.176 & Var & 29.98 \\
\hline & $\mathrm{R}$ & 43 & 3.87 & 2.215 & Var & 19.82 \\
\hline & $\mathrm{I}$ & 42 & 1.558 & 2.236 & $\mathrm{NV}$ & - \\
\hline \multirow[t]{4}{*}{27.09 .2016} & B & 42 & 2.113 & 2.236 & NV & - \\
\hline & $\mathrm{V}$ & 42 & 0.864 & 2.236 & NV & - \\
\hline & $\mathrm{R}$ & 41 & 0.779 & 2.258 & NV & - \\
\hline & I & 37 & 0.362 & 2.358 & NV & - \\
\hline \multirow[t]{4}{*}{28.09 .2016} & B & 50 & 1.284 & 2.091 & $\mathrm{NV}$ & - \\
\hline & $\mathrm{V}$ & 46 & 1.002 & 2.157 & NV & - \\
\hline & $\mathrm{R}$ & 47 & 1.387 & 2.14 & NV & - \\
\hline & I & 49 & 0.567 & 2.106 & NV & - \\
\hline \multirow[t]{4}{*}{29.09 .2016} & B & 49 & 1.088 & 2.106 & $\mathrm{NV}$ & - \\
\hline & $\mathrm{V}$ & 49 & 1.959 & 2.106 & NV & - \\
\hline & $\mathrm{R}$ & 50 & 1.115 & 2.091 & NV & - \\
\hline & $\mathrm{I}$ & 42 & 1.125 & 2.236 & $\mathrm{NV}$ & - \\
\hline \multirow[t]{4}{*}{30.09 .2016} & B & 38 & 1.898 & 2.331 & $\mathrm{NV}$ & - \\
\hline & $\mathrm{V}$ & 35 & 0.330 & 2.416 & NV & - \\
\hline & $\mathrm{R}$ & 34 & 0.429 & 2.448 & NV & - \\
\hline & I & 36 & 0.853 & 2.386 & NV & - \\
\hline
\end{tabular}

$F=$ Enhanced $F$-test values; $F_{c}(0.001)=$ Critical Values of $F$ Distribution at $0.1 \%$; Amplitude: Variability Amplitude Var/NV: Variable/Non-variable. 


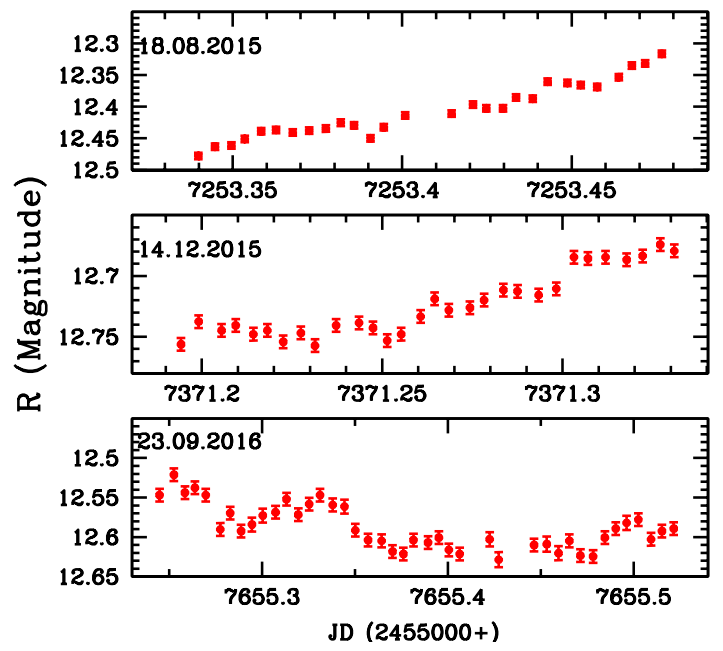

Figure 3. Typical examples of LCs of BL Lacertae where $\Delta \mathrm{m}>0.10$ in a few hours.

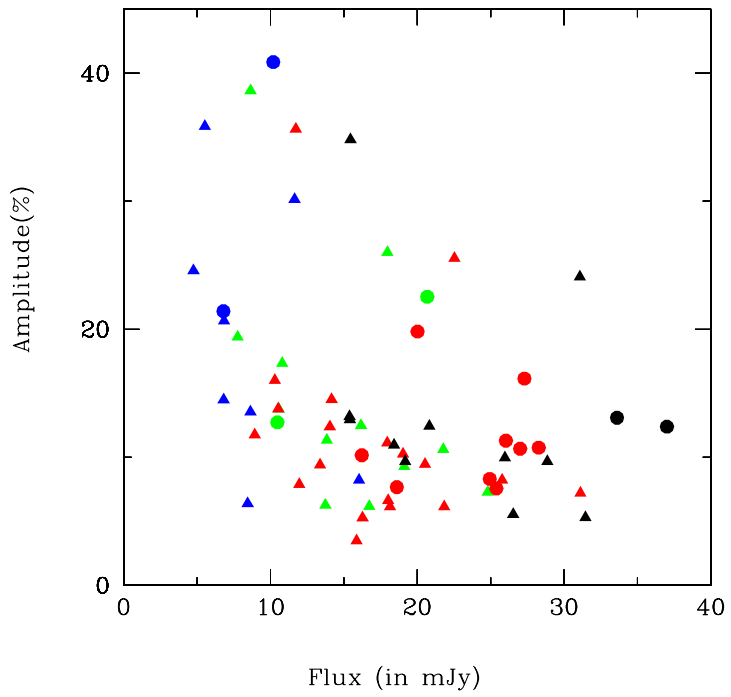

Figure 4. Amplitude of variability versus flux. Again, blue, green, red and black colors represent $\mathrm{B}, \mathrm{V}, \mathrm{R}$ and I bands, respectively, triangles give data from [19] and circles provide these new data. The Spearman correlation coefficient is -0.42 with $p>0.9996$.

The inter-band time lags between the B, V, R and I bands for all of the LCs presented here were computed using Discrete Correlation Functions (DCF; details are given in [19]). All the lags are consistent with zero, as expected because the optical bands are very close to each other; if lags are present, they must be shorter than the resolution in our LCs, which is $\sim 8 \mathrm{~min}$.

\subsection{Color Indices}

We searched for spectral variations in BL Lac by looking at how color varied with respect to the source brightness. We calculated color indices (CIs) by combining nearly simultaneous (within 8 min) V, R and I measurements to produce CIs: V-R and R-I.

It should be noted that the light from the host galaxy or the accretion disk in the AGN can also affect the color variations of BL Lac [37]. Hence, in calculating the color indices, we have used data that is corrected for the host galaxy contribution. In addition, since the source is in a bright state during our observations, the accretion disk component can be ignored, as the Doppler boosted flux from the relativistic jet would dominate the overall emission. 
We found significant correlations between CI and magnitude in 24 of our observations. One example is shown in Figure 5. To quantify this, we follow [19] and fit the color-R-magnitude diagrams with a linear model of the form CI $=m \times$ mag $+c$. Pearson correlation coefficients $(r)$ and their $p$-values (here, the null hypothesis probability, where we take a confirmed CI correlation with the $\mathrm{R}$ magnitude when $p<0.05$ ) as well as the values of slopes, $m$, and intercepts, $c$ are given in Table 3 .

In these plots of 24 of our observations, the CIs positively correlate with the source brightness, which shows hardening of the spectrum as BL Lac gets brighter (or a bluer-when-brighter behavior). In the remaining 19 observations where we had collected sufficient data to search for CI-brightness trends, we did not find any significant correlations. These could be explained by the lighthouse effect in the shock-in-jet model (e.g., [38]), where relativistic electrons are moving towards an observer on helical trajectories in the jets and the flares will be produced by the sweeping beam whose direction varies with time but no differences in variations can be seen over such limited ranges in frequency.

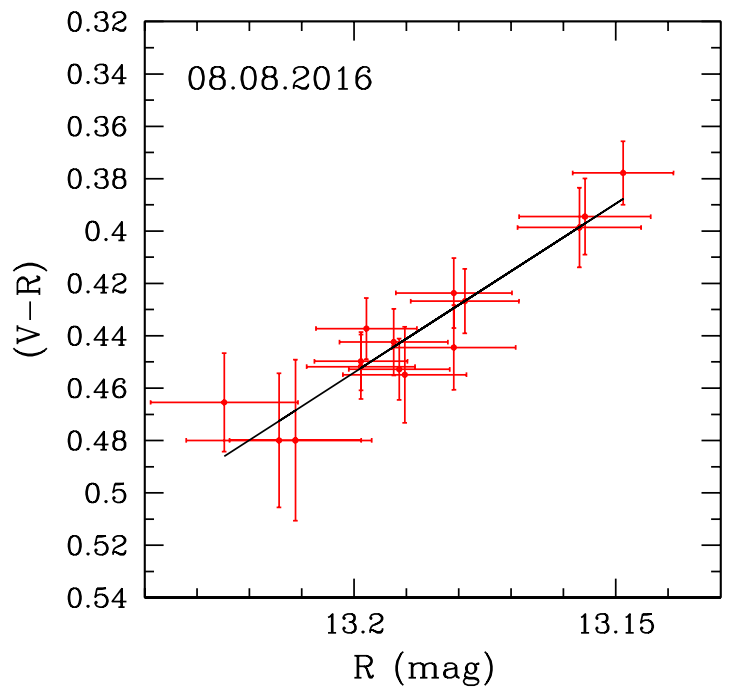

Figure 5. Sample color-magnitude diagram for BL Lacertae.

Table 3. Results of linear fits to color index-flux diagrams.

\begin{tabular}{cccccc}
\hline $\begin{array}{c}\text { Date of Observation } \\
\text { dd.mm.yyyy }\end{array}$ & Band & $\boldsymbol{r}$ & $\boldsymbol{p}$ & $\boldsymbol{m}$ Slope & $\boldsymbol{c}$ Intercept \\
\hline 19.07 .2015 & $(\mathrm{R}-\mathrm{I})$ & 0.65 & 0.0002 & 0.42 & -4.76 \\
20.07 .2015 & $(\mathrm{R}-\mathrm{I})$ & 0.51 & 0.011 & 0.39 & -4.31 \\
21.07 .2015 & $(\mathrm{R}-\mathrm{I})$ & 0.53 & 0.015 & 0.22 & -2.18 \\
22.07 .2015 & $(\mathrm{R}-\mathrm{I})$ & 0.41 & 0.047 & 0.41 & -4.54 \\
18.08 .2015 & $(\mathrm{R}-\mathrm{I})$ & 0.62 & 0.0005 & 0.39 & -4.23 \\
27.08 .2015 & $(\mathrm{R}-\mathrm{I})$ & 0.88 & $3.74 \times 10^{-7}$ & 1.13 & -14.10 \\
08.09 .2015 & $(\mathrm{R}-\mathrm{I})$ & 0.42 & 0.004 & 0.29 & -3.10 \\
14.09 .2015 & $(\mathrm{R}-\mathrm{I})$ & 0.73 & $3.51 \times 10^{-5}$ & 0.87 & -10.52 \\
15.09 .2015 & $(\mathrm{R}-\mathrm{I})$ & 0.66 & 0.00012 & 0.42 & -4.60 \\
05.11 .2015 & $(\mathrm{R}-\mathrm{I})$ & 0.41 & 0.026 & 0.47 & -5.26 \\
06.11 .2015 & $(\mathrm{R}-\mathrm{I})$ & 0.46 & 0.009 & 0.15 & -1.23 \\
07.11 .2015 & $(\mathrm{R}-\mathrm{I})$ & 0.60 & 0.0002 & 0.80 & -9.38 \\
11.11 .2015 & $(\mathrm{R}-\mathrm{I})$ & 0.33 & 0.028 & 0.14 & -1.12 \\
12.11 .2015 & $(\mathrm{R}-\mathrm{I})$ & 0.35 & 0.023 & 0.76 & -8.92 \\
03.12 .2015 & $(\mathrm{R}-\mathrm{I})$ & 0.72 & 0.004 & 0.73 & -8.60 \\
30.06 .2016 & $(\mathrm{R}-\mathrm{I})$ & 0.45 & 0.045 & 0.66 & -7.72 \\
08.08 .2016 & $(\mathrm{~V}-\mathrm{R})$ & 0.94 & $1.83 \times 10^{-7}$ & 1.29 & -16.58 \\
26.08 .2016 & $(\mathrm{~V}-\mathrm{R})$ & 0.65 & 0.0004 & 0.54 & -6.94 \\
30.08 .2016 & $(\mathrm{R}-\mathrm{I})$ & 0.33 & 0.017 & 0.21 & -2.26 \\
\hline
\end{tabular}


Table 3. Cont.

\begin{tabular}{cccccc}
\hline $\begin{array}{c}\text { Date of Observation } \\
\text { dd.mm.yyyy }\end{array}$ & Band & $\boldsymbol{r}$ & $\boldsymbol{p}$ & $\boldsymbol{m}$ Slope & $\boldsymbol{c}$ Intercept \\
\hline 22.09 .2016 & $(\mathrm{R}-\mathrm{I})$ & 0.68 & 0.016 & 0.46 & -5.21 \\
24.09 .2016 & $(\mathrm{R}-\mathrm{I})$ & 0.66 & 0.0009 & 0.44 & -5.02 \\
27.09 .2016 & $(\mathrm{R}-\mathrm{I})$ & 0.55 & 0.0004 & 0.47 & -5.51 \\
28.09 .2016 & $(\mathrm{R}-\mathrm{I})$ & 0.72 & $8.30 \times 10^{-9}$ & 0.50 & -5.79 \\
30.09 .2016 & $(\mathrm{R}-\mathrm{I})$ & 0.65 & $3.03 \times 10^{-5}$ & 0.51 & -5.96 \\
\hline$r$ and $p$ : Pearson Correlation Coefficient and its null hypothesis probability values, respectively.
\end{tabular}

BL Lacertae does sometimes exhibit bluer-when-brighter trends with a range of regression slopes but no redder-when-brighter behavior. This bluer-when-brighter dominance for BL Lac has been reported earlier on both long-term and short-term time-scales (e.g., [21,39-43]). Villata 2004 [39] characterized intra-day flares to be strongly bluer-when-brighter chromatic events with a slope of $\sim 0.4$. They noted that these could be modeled if there was one variable component with a flatter slope that was dominant during the flaring states as well as a steeper sloped component that was more stable and produces the bulk of the longer term, basically achromatic, emission. In our current study, we found bluer-when-brighter changes in CI with slopes that were in the range 0.14-1.13 (Table 3). Hence, we find that the intra-night flares seen during 2014-2016 are usually more chromatic as compared to our earlier studies for the period 2010-2012 during where significant positive slopes only ranged between $0.14-0.29$ [19].

In some of the light curves, significant color variations are seen, but the slopes are relatively flat, such as for the observations performed on 11 November 2015, 18 August 2015, 21 July 2015, and 30 August 2016. This could be explained by differences beween flux states during these particular observations. It can be seen from the LCs of these observations that they exhibit more small sub-flares on top of the long-term rising or decaying trends. Hence, the superposition of multiple components can produce an overall weakening of the color-magnitude relations. Similar findings were made by [33], where they found that their sample of BL Lacs showed complicated color-magnitude diagram behavior including hysteresis tracks, as well as achromatic flares; these indicate that different jet components dominate at different times. Dai [44] found intra-day spectral hysteresis loop patterns for the blazar S5 $0716+714$ in its color-magnitude diagram. These loop-like patterns are attributed to the differences in the amplitudes and cadences at different wavelengths [18]. We did not find any hints of such loop patterns during our observations, given the absence of any detectable time lags between bands.

\section{Discussion}

We have presented our photometric monitoring of BL Lacertae during three seasons in 2014-2016 for a total of 43 nights in the B, V, R and I bands in order to further study its flux and spectral variability. We found genuine IDV in a total of 14 nights of observation using the power enhanced F-test at a stringent level. The LCs frequently exhibit gradual rises and decays, sometimes with superimposed sub-flares. We found no evidence for periodicity or other characteristic time scales. Various models that can explain optical IDV of blazars involve: changes in the electron energy density distribution of the relativistic particles that produce variable synchrotron emission; shocks accelerating particles in the bulk relativistic plasma jet; turbulence behind an outgoing shock in a jet; irregularities in the jet flows, such as "mini-jets" (e.g., [15,28-30,45,46]). As we are focused on intra-night flux variations that involve color variations, these are very likely to be associated with models centered on shocks moving through a turbulent plasma jet.

We found the duty cycle of the source between 2014 and 2016, based on the light curves of all the bands to be $\sim 12 \%$, which is low compared to the DC (44\%) found in our previous campaign during 2010-2012 over 38 nights of observations. If we restrict consideration to the $\mathrm{R}$ band, only the current DC is $\sim 25 \%$ while that for the earlier observations was $\sim 49 \%$. BL Lacertae is an LBL and previous 
studies have indicated that LBLs typically display stronger IDV than do HBLs (high frequency peaked blazars). The DCs have been claimed to be as high as $\sim 70 \%$ for LBLs and $\sim 30-50 \%$ for HBLs $([47,48])$ However, Ref. [49] found that the overall DC was $22 \%$ for HBLs and 50\% for LBLs in a large sample of blazars. Recently, [50] considered a group of LBLs in X-ray bands and found that the since the peaks of the synchrotron component for such sources lie in the near IR/optical, more energetic electrons are available for the synchrotron emission there and hence they display more DC in these bands. Hence, a DC of $\sim 44 \%$ for BL Lacertae is in agreement with previous studies, but the current $\sim 12 \%$ value is low. This could well be related to the overall high state of BL Lac during our recent observations. However, it has been found that, during this period, BL Lacertae has not shown any flaring activity at radio frequencies ([51,52]).

The variability amplitude is typically larger at higher frequencies. This is generally explained in terms of higher energy electrons only being able to emit closer to the shock front as those more quickly lose energy while producing higher frequency synchrotron radiation [53]. Due to the closeness of the B, V, R and I bands, the time when a flare is initiated and their lengths are nearly the same for all optical bands. The errors in our B band measurements for this campaign are higher than those in lower frequency bands, hence on many occasions the amplitude of variability is not seen at sufficient statistical strength, and generally not seen as stronger, at higher frequencies. We found significant positive correlations between the observed variability amplitude with respect to the length of the nightly observations. It can be seen from Figures 1 and 2 that the duration of the monitoring of our observations varies between $1.5-7 \mathrm{~h}$.

We found that the probability of seeing a significant intra-day variability usually increases if the source is continuously observed for more than $4 \mathrm{~h}$, but most of our measurements were shorter than $4 \mathrm{~h}$. We also searched for correlations between the variability amplitude with respect to flux and saw that the amplitude drops as the source flux rises. This trend could be explained if, as the source flux increases, the fluctuations in the turbulent jet decrease [28] and thus this more uniform jet flow leads to a decline in the variability amplitude.

We looked for correlations between magnitude and color-index, finding significant (>95\%) positive correlations in 24 observations out of the total of 43. BL Lac exhibited bluer-when-brighter trends with slopes that varied between $0.14-1.13$ as shown in Table 3. We never saw redder-whenbrighter behavior.

The structure of color-magnitude diagrams gives information on the amplitude differences and time-lags between the observed bands. As the optical bands have close proximity, it is often hard to measure time lags between them, as was the case for these observations. The simulations of [54] indicated that both the amplitude differences and time delays between temporal changes at different wavelengths typically result in spectral hardening while a flare rises. They showed that the amplitude difference between two light curves resulted in a diagonal path in the color-magnitude diagram, whereas a time-lag combined with amplitude variations resulted in a counter-clockwise loop pattern on a color index-magnitude plot. As we were not able to determine any significant lags between these bands, we can not address this possibility and can only conclude that any lags, if present, are probably shorter than the sampling time of our observations.

\section{Conclusions}

Our conclusions can be summarized as follows:

- We observed BL Lacertae quasi-simultaneously in B, V, R and I bands during the period 2014-2016 in 43 nights to search for intra-day variability and found strong variability in at least one band on a total of 14 nights.

- BL Lac was moderately variable with changes well correlated across the bands. The variations usually were smooth, with gradual rises/decays. Magnitude changes of over $\sim 0.10$ over a few hours were seen on several nights. 
- The amplitude of variability is positively correlated with the duration of the observation, but it decreases as the source becomes brighter.

- We did not find any significant time delays between the B, V, R and I bands in these observations. This indicates that the variations are nearly simultaneous across the optical bands and any possible time lags are shorter than our data sampling interval of $\sim 8 \mathrm{~min}$.

- The flux changes are accompanied by spectral variations on intra-day time-scales. In 24 of the 43 observations, the optical spectrum showed the overall bluer-when-brighter trend, probably associated with very variable jet emission.

- The color vs. magnitude diagrams are highly chromatic on the intra-night timescales and are likely associated with the rapid injection or acceleration of relativistic particles, subsequently followed by rapid synchrotron cooling.

Acknowledgments: We thank the referees for constructive comments that have improved the presentation of our work. H.G. is sponsored by the Chinese Academy of Sciences (CAS) Visiting Fellowship for Researchers from Developing Countries; CAS Presidents International Fellowship Initiative (PIFI) (Grant No. 2014FFJB0005); supported by the National Natural Science Foundation of China (NSFC) Research Fund for International Young Scientists (Grant Nos. 11450110398, 11650110434) and supported by a Special Financial Grant from the China Postdoctoral Science Foundation (Grant No. 2016T90393). This research was partially supported by the Bulgarian National Science Fund of the Ministry of Education and Science under grants DN 08-1/2016 and DM08-2/2016 and by funds of the project RD-08-102 of Shumen University. The authors thank the Director of Skinakas Observatory Ioannis Papamastorakis and Iossif Papadakis for the award of telescope time. The Skinakas Observatory is a collaborative project of the University of Crete, the Foundation for Research and Technology-Hellas, and the Max-Planck-Institut für Extraterrestrische Physik. M.F.G. acknowledges support from the National Science Foundation of China (Grant 11473054 and U1531245) and by the Science and Technology Commission of Shanghai Municipality (14ZR1447100).

Author Contributions: H.G. and A.C.G. planned the project. H.G. did major work e.g., further analysis of the intra-day light curves, made the plots, and wrote the manuscript. A.C.G., P.J.W. and M.F.G. contributed to the writing and interpretation of the results of the manuscript. R.B., A.S., E.S., and S.I. performed observations and photometric analysis of the data.

Conflicts of Interest: The authors declare no conflict of interest.

\section{References}

1. Miller, J.S.; Hawley, S.A. The Spectrum and Redshift of BL Lacertae. Astrophys. J. Lett. 1977, 212, L47-L50.

2. Wagner, S.J.; Witzel, A. Intraday Variability in Quasars and BL Lac Objects. Annu. Rev. Astron. Astrophys. 1995, 33, 163-198.

3. Vermeulen, R.C.; Ogle, P.M.; Tran, H.D.; Browne, I.W.A.; Cohen, M.H.; Readhead, A.C.S.; Taylor, G.B.; Goodrich, R.W. When Is BL Lac Not a BL Lac? Astrophys. J. Lett. 1995, 452, L5-L8.

4. Raiteri, C.M.; Villata, M.; Capetti, A.; Aller, M.F.; Bach, U.; Calcidese, P.; Gurwell, M.A.; Larionov, V.M.; Ohlert, J.; Nilsson, K.; et al. WEBT multiwavelength monitoring and XMM-Newton observations of BL Lacertae in 2007-2008. Unveiling different emission components. Astron. Astrophys. 2009, 507, 769-779.

5. Böttcher, M.; Marscher, A.P.; Ravasio, M.; Villata, M.; Raiteri, C.M.; Aller, H.D.; Aller, M.F.; Teräsranta, H.; Mang, O.; Tagliaferri, G.; et al. Coordinated Multiwavelength Observations of BL Lacertae in 2000. Astrophys. J. 2003, 596, 847-859.

6. Villata, M.; Raiteri, C.M.; Larionov, V.M.; Nikolashvili, M.G.; Aller, M.F.; Bach, U.; Carosati, D.; Hroch, F.; Ibrahimov, M.A.; Jorstad, S.G.; et al. The correlated optical and radio variability of BL Lacertae. WEBT data analysis 1994-2005. Astron. Astrophys. 2009, 501, 455-460.

7. Raiteri, C.M.; Villata, M.; Bruschini, L.; Capetti, A.; Kurtanidze, O.M.; Larionov, V.M.; Romano, P.; Vercellone, S.; Agudo, I.; Aller, H.D.; et al. Another look at the BL Lacertae flux and spectral variability. Observations by GASP-WEBT, XMM-Newton, and Swift in 2008-2009. Astron. Astrophys. 2010, 524, A43.

8. Raiteri, C.M.; Villata, M.; D'Ammando, F.; Larionov, V.M.; Gurwell, M.A.; Mirzaqulov, D.O.; Smith, P.S.; Acosta-Pulido, J.A.; Agudo, I.; Arévalo, M.J.; et al. The awakening of BL Lacertae: Observations by Fermi, Swift and the GASP-WEBT. Mon. Not. R. Astron. Soc. 2013, 436, 1530-1545.

9. Massaro, E.; Nesci, R.; Maesano, M.; Montagni, F.; D’Alessio, F. Fast variability of BL Lacertae at $1 \mathrm{~m}$. Mon. Not. R. Astron. Soc. Lett. 1998, 299, 47-50. 
10. Tosti, G.; Luciani, M.; Fiorucci, M.; Maesano, M.; Massaro, E.; Montagni, F.; Nesci, R.; Kurtanidze, O.; Nikolashvili, M.; Natriashvili, V.; et al. The Optical Light curve of BL Lacertae during the 1997 outburst. Blazar Data 1999, 2, 1.

11. Clements, S.D.; Carini, M.T. Multiband Microvariability Observations of BL Lacertae during the Outburst of 1997. Astron. J. 2001, 121, 90-96.

12. Hagen-Thorn, V.A.; Larionov, V.M.; Larionova, E.G.; Kudryavtseva, N.A.; Tikhonov, A.V.; Hagen-Thorn, A.V.; Arkharov, A.A.; D'Alessio, F. VizieR Online Data Catalog: BV (RI) c-JHK photometry of BL Lac in 1999-2001 (Hagen-Thorn+, 2004). Astron. Lett. 2004, 903, 209-217.

13. Agarwal, A.; Gupta, A.C. Multiband optical variability studies of BL Lacertae. Mon. Not. R. Astron. Soc. 2015, 450, 541-551.

14. Marscher, A.P.; Jorstad, S.G.; D'Arcangelo, F.D.; Smith, P.S.; Williams, G.G.; Larionov, V.M.; Oh, H.; Olmstead, A.R. The inner jet of an active galactic nucleus as revealed by a radio-to- $\gamma$-ray outburst. Nature 2008, 452, 966-969.

15. Gaur, H.; Gupta, A.C.; Wiita, P.J.; Uemura, M.; Itoh, R.; Sasada, M. Anti-correlated Optical Flux and Polarization Variability in BL Lac. Astrophys. J. Lett. 2014, 781, L4.

16. Villata, M.; Raiteri, C.M.; Kurtanidze, O.M.; Nikolashvili, M.G.; Ibrahimov, M.A.; Papadakis, I.E.; Tsinganos, K.; Sadakane, K.; Okada, N.; Takalo, L.O.; et al. The WEBT BL Lacertae Campaign 2000. Astron. Astrophys. 2002, 390, 407-421.

17. Papadakis, I.E.; Boumis, P.; Samaritakis, V.; Papamastorakis, J. Multi-band optical micro-variability observations of BL Lacertae. Astron. Astrophys. 2003, 397, 565-573.

18. Hu, S.M.; Wu, J.H.; Zhao, G.; Zhou, X. Optical multiband observations of BL Lacertae during the outburst of 2005. Mon. Not. R. Astron. Soc. Lett. 2006, 373, 209-216.

19. Gaur, H.; Gupta, A.C.; Bachev, R.; Strigachev, A.; Semkov, E.; Böttcher, M.; Wiita, P.J.; de Diego, J.A.; Gu, M.F.; Guo, H.; et al. Nature of intranight optical variability of BL Lacertae. Mon. Not. R. Astron. Soc. 2015, 452, 4263-4273.

20. Gaur, H.; Gupta, A.C.; Bachev, R.; Strigachev, A.; Semkov, E.; Wiita, P.J.; Volvach, A.E.; Gu, M.F.; Agarwal, A.; Agudo, I.; et al. Optical and radio variability of BL Lacertae. Astron. Astrophys. 2015, 582, A103.

21. Gaur, H.; Gupta, A.C.; Strigachev, A.; Bachev, R.; Semkov, E.; Wiita, P.J.; Peneva, S.; Boeva, S.; Kacharov, N.; Mihov, B.; et al. Quasi-simultaneous two-band optical variability of the blazars 1ES $1959+650$ and 1ES 2344+514. Mon. Not. R. Astron. Soc. 2012, 420, 3147-3162.

22. Villata, M.; Raiteri, C.M.; Lanteri, L.; Sobrito, G.; Cavallone, M. BVR photometry of comparison stars in selected blazar fields. I. Photometric sequences for 10 BL Lacertae objects. Astron. Astrophys. Suppl. 1998, 130, 305-310.

23. De Diego, J.A. Testing Tests on Active Galactic Nucleus Microvariability. Astron. J. 2010, 139, 1269-1282.

24. De Diego, J.A. On the Reliability of Microvariability Tests in Quasars. Astron. J. 2014, 148, 93.

25. De Diego, J.A.; Polednikova, J.; Bongiovanni, A.; García, A.M.P.; De Leo, M.A.; Verdugo, T.; Cepa, J. Testing Microvariability in Quasar Differential Light Curves Using Several Field Stars. Astron. J. 2015, 150, 44.

26. Romero, G.E.; Cellone, S.A.; Combi, J.A. Optical microvariability of southern AGNs. Astron. Astrophys. Suppl. 1999, 135, 477-486.

27. Stalin, C.S.; Sagar, R.; Wiita, P.J. Optical Variability Properties of High Luminosity AGN Classes. J. Astrophys. Astron. 2004, 25, 1-55.

28. Marscher, A.P. Turbulent, Extreme Multi-zone Model for Simulating Flux and Polarization Variability in Blazars. Astrophys. J. 2014, 780, 87.

29. Calafut, V.; Wiita, P.J. Modeling the Emission from Turbulent Relativistic Jets in Active Galactic Nuclei. J. Astrophys. Astron. 2015, 36, 255-268.

30. Pollack, M.; Pauls, D.; Wiita, P.J. Variability in Active Galactic Nuclei from Propagating Turbulent Relativistic Jets. Astrophys. J. 2016, 820, 12.

31. Heidt, J.; Wagner, S.J. Statistics of optical intraday variability in a complete sample of radio-selected BL Lacertae objects. Astron. Astrophys. 1996, 305, 42-52.

32. Ghisellini, G.; Villata, M.; Raiteri, C.M.; Bosio, S.; de Francesco, G.; Latini, G.; Maesano, M.; Massaro, E.; Montagni, F.; Nesci, R.A.; et al. Optical-IUE observations of the gamma-ray loud BL Lacertae object S5 0716+714: Data and interpretation Astron. Astrophys. 1997, 327, 61-71 
33. Bonning, E.; Urry, C.M.; Bailyn, C.; Buxton, M.; Chatterjee, R.; Coppi, P.; Fossati, G.; Isler, J.; Maraschi, L. SMARTS Optical and Infrared Monitoring of 12 Gamma-Ray Bright Blazars. Astrophys. J. 2012, 756, A13.

34. Ghosh, K.K.; Ramsey, B.D.; Sadun, A.C.; Soundararajaperumal, S. Optical Variability of Blazars. Astrophys. J. Suppl. 2000, 127, 11-26.

35. Ramírez, A.; de Diego, J.A.; Dultzin-Hacyan, D.; González-Pérez, J.N. Optical variability of PKS $0736+017$. Astron. Astrophys. 2004, 421, 83-89.

36. Gupta, A.C.; Cha, S.-M.; Lee, S.; Jin, H.; Pak, S.; Cho, S-H.; Moon, B.; Park, Y.; Yuk, I.-S.; Nam, U.-W.; et al. Multicolor Near-Infrared Intra-Day and Short-Term Variability of the Blazar S5 0716+714. Astron. J. 2008, 136, 2359-2366.

37. Hawkins, M.R.S. Quasar Variability: New Surveys and New Models. IAU Colloquium 184. ASP Conf. Proc. 2004, 284, 351.

38. Wiita, P.J. Swinging jets and the variability of active galactic nuclei. Astron. Astrophys. 1992, 259, $109-117$.

39. Villata, M.; Raiteri, C.M.; Kurtanidze, O.M.; Nikolashvili, M.G.; Ibrahimov, M.A.; Papadakis, I.E.; Tosti, G.; Hroch, F.; Takalo, L.O.; Sillanpää, A.; et al. The WEBT BL Lacertae Campaign 2001 and its extension. Optical light curves and colour analysis 1994-2002. Astron. Astrophys. 2004, 421, 103-114.

40. Stalin, C.S.; Sagar, R.; Wiita, P.J.; Mohan, V.; Pandey, A.K. Multiband optical monitoring of the blazars S5 0716+714 and BL Lacertae. Mon. Not. R. Astron. Soc. 2006, 366, 1337-1345.

41. Gu, M.F.; Lee, C.-U.; Pak, S.; Yim, H.S.; Fletcher, A.B. Multi-colour optical monitoring of eight red blazars. Astron. Astrophys. 2006, 450, 39-51.

42. Larionov, V.M.; Villata, M.; Raiteri, C.M. The nature of optical and near-infrared variability of BL Lacertae. Astron. Astrophys. 2010, 510, A93.

43. Wierzcholska, A.; Ostrowski, M.; Stawarz, L.; Wagner, S.; Hauser, M. Longterm optical monitoring of bright BL Lacertae objects with ATOM: Spectral variability and multiwavelength correlations. Astron. Astrophys. 2015, 573, A69.

44. Dai, Y.; Wu, J.; Zhu, Z.-H.; Zhou, X.; Ma, J.; Yuan, Q.; Wang, L. Seven-year Multi-color Optical Monitoring of BL Lacertae Object S5 0716+714. Astrophys. J. Suppl. 2013, 204, 22.

45. Marscher, A.P.; Gear, W. K.; Travis, J.P. Variability of Nonthermal Continuum Emission in Blazars. Var. Blaz. 1992, 85, 87 .

46. Giannios, D.; Uzdensky, D.A.; Begelman, M.C. Fast TeV variability in blazars: Jets in a jet. Mon. Not. R. Astron. Soc. Lett. 2009, 395, L29-L33.

47. Heidt, J.; Wagner, S.J. Intraday variability in X-ray selected BL Lacertae objects. Astron. Astrophys. 1998, 329, 853-862.

48. Romero, G.E.; Cellone, S.A.; Combi, J.A.; Andruchow, I. Optical microvariability of EGRET blazars. Astron. Astrophys. 2002, 390, 431-438.

49. Goyal, A.; Joshi, S.; Karthick, C.; Sagar, R.; Wiita, P.J.; Anupama, G.C.; Sahu, D.K. Rapid optical variability of TeV blazars. Mon. Not. R. Astron. Soc. 2011, 416, 101-117.

50. Gupta, A.C.; Kalita, N.; Gaur, H.; Duorah, K. Peak of spectral energy distribution plays an important role in intra-day variability of blazars? Mon. Not. R. Astron. Soc. Lett. 2016, 462, 1508-1516.

51. Tsujimoto, S.; Acosta, M.V.; Lindfors, E.; Mazin, D.; Pedaletti, G.; Ramazani, V.F.; D'Ammand, F.; Sitarek, J.; Kushida, J.; Nishijima, K. Multiwavelength observations of the blazar BL Lacertae in June 2015. arXiv 2017, arXiv:1709.05063.

52. Kim, D.W.; Trippe, S.; Lee, S.S.; Park, J.H.; Kim, J.Y.; Algaba, J.C.; Hodgson, J.A.; Kino, M.; Zhao, G.Y.; Wajima, K.; et al. The Millimeter-Radio Emission of BL Lacertae During Two gamma-ray Outbursts. arXiv 2017, arXiv:1711.05952.

53. Marscher, A.P.; Gear, W.K. Models for high-frequency radio outbursts in extragalactic sources, with application to the early 1983 millimeter-to-infrared flare of 3C 273. Astrophys. J. 1985, 298, 114-127.

54. Dai, Y.; Wu, J.; Zhu, Z.-H.; Zhou, X.; Ma, J. Color Behavior of BL Lacertae Object OJ 287 during an Optical Outburst. Astron. J. 2011, 141, 65.

(C) 2017 by the authors. Licensee MDPI, Basel, Switzerland. This article is an open access article distributed under the terms and conditions of the Creative Commons Attribution (CC BY) license (http://creativecommons.org/licenses/by/4.0/). 